\title{
Fundamental study of mechanism of band gap in fluid and solid/fluid phononic crystals Eric $\mathrm{Li}^{2}, \mathrm{ZC} \mathrm{He}^{1 *}, \mathrm{G}$ Wang ${ }^{1}$, Yong Jong ${ }^{3}$ \\ ${ }^{1}$ State Key Laboratory of Advanced Design and Manufacturing for Vehicle Body, Hunan University, Changsha, 410082 P. R. China \\ ${ }^{2}$ School of Science, Engineering \& Design, Teesside University, Middlesbrough, UK \\ ${ }^{3}$ Hefei General Machinery Research Institute, No. 888, West Changjiang Road, Hefei, Anhui 230031, China
}

\begin{abstract}
Phononic crystals (PCs) have possessed outstanding features to control/manipulate the propagation of the acoustic/sound wave. In this paper, the local resonant elements, such as local resonant cavity in fluid PCs and local resonant inclusion in solid/fluid PCs, are introduced. The effect of geometry parameters, Poisson's ratio, Young's modulus on the band gap solid/fluid PCs are investigated in detail. It is found that wider multiple band gaps are obtained for the fluid PCs with local resonant cavity of "+" hole compared with square and circle holes. More importantly, the very low-frequency band gaps can be obtained by introducing the local resonant inclusion with consideration of fluid-structural interaction for solid/fluid PCs. In addition, we have compared the sound transmission loss in fluid and solid/fluid PCs. The numerical results have clearly indicated that solid/fluids PCs with consideration of fluid-structural interaction can block the propagation of stress wave effectively compared with fluid PCs. The theoretical study and numerical simulation conducted in this work have provided a new avenue to design more innovative fluid and solid/fluid PCs.
\end{abstract}

Key words: phononic crystal; band gap; fluid-structural interaction; transmission loss

${ }^{\text {" }}$ State Key Laboratory of Advanced Design and Manufacturing for Vehicle Body, Hunan University, Changsha, 410082 P. R. China, Email address: hezhicheng815@hnu.edu.cn 


\section{Introduction}

Phononic crystals (PCs) are artificial materials with many appealing properties that cannot be found in nature, which has received substantial attention in the literature [1-6]. The idea of PCs is originated from electromagnetic metamaterials with simultaneously negative electric permittivity and magnetic permeability[7]. With combination of periodic two or three more base materials with different microstructures, the overall performance of PCs can be tailored by appropriate choices of changing the microscopic size and configuration of the individual constituents instead of chemical components. The potential applications of PCs include acoustic cloaking [8], fluid sensor [9], imaging [10], energy harvesting $[11,12]$ and sound attenuation [6]. In doing so, the design methodology of PCs has explored an entirely new route to develop more innovative materials and structure.

The most promising feature of PCs is the appearance of band gap [13], within which the elastic/acoustic waves are prohibited in all directions. Such unique but very useful characteristic of PCs is extremely important in the practical application of control of noise and vibration. Generally, there are two types of working mechanics to create the band gap of PCs, which include Bragg scattering and local resonance. It is noted that Bragg band gaps usually exist in the frequency region where the wavelength of the waves is in the same order of magnitude with the lattice constant, which limits the practical application of PCs in low-frequency region [14]. To this end, Liu et al. developed locally resonant sonic materials (LRSMs), which is made of spherical lead inclusions with a rubber coating and embedded in an epoxy matrix. Both experiment and theory validated that the lattice constant of PCs could be much smaller than the longitudinal wavelength of the wave in epoxy in the creation of low frequency band gap [15]. Consequently, Wang et al. proposed 
two dimensional binary locally resonant phononic crystals, which are composed of periodic soft rubber cylinders in epoxy host [16].

The tuning of band gap of PCs is always a very active research area. In the past, a large amount of research has been done to study the relation between the band gap and scatterer properties such as sizes, shapes, and lattice structures [17]. Liu et al. [17] have comprehensively studied the effect of the pore shapes on the band gap of PCs. In addition, the influence of release holes on the band gaps of solid-solid PCs was investigated by Soliman et al [18]. Feng et al. [19, 20] demonstrated that the band gaps of finite PCs could be tuned by the initial stress. The band gaps are found to be determined by several factors, including material and geometry parameters. Wang el al. studied the band gap properties of two-dimensional PCs with cross-like holes using the finite element method (FEM) [21]. Based on the theoretical study and numerical simulation, it is observed that non- convex ("+" geometry) provides wider and lower band gaps compared with convex geometry such as square and circular holes. However, the past analysis of band gap with non-convex hole was only focused in solid/ solid PCs.

In general, we can define fluid/fluid, solid/solid, fluid/solid and solid/ fluid PCs [22]. In the fluid PCs, longitudinal wave in the fluid PCs exists, and the fluid PCs offers an innovative platform for acoustic fluid sensors that are still in the earlier stage development [23]. It is well known that mixed transverse and longitudinal waves with different velocities propagate within the solids, while only the longitudinal (acoustic) mode exists in ideal fluids [24]. However, the fluid-structural interaction [25, 26] must be taken into account at the interface between the solid and fluid in the analysis of solid/fluid PCs [27, 28], which makes it difficult predict the physical responses of PCs with solid and fluid 
components. Furthermore, the mechanism of band gap in the solid/fluid PCs with consideration of fluid-structural interaction is still not fully investigated.

In parallel with theoretical studies of PCs, modeling and simulation is also crucial to advance the future development of PCs. As a reliable, robust and effective approach, FEM is extensively employed to study the acoustic and elastic waves in periodic PCs. Compared with finite difference method (FDM), FEM is very powerful to deal with complex geometry efficiently. In 2004, Wang et al. proposed a lumped-mass FEM with faster convergence rate to analyze the propagations of elastic wave in two dimensional PCs [29]. Liu and Gao developed an explicit dynamic FEM to compute the band-structure of 2D PCs [30]. Furthermore, the X-extended Finite Element Method (X-FEM) was formulated to compute the band structure of 3D mechanical metamaterials with complicated geometry [31]. Consequently, Li et al proposed different types of smoothed finite element method (SFEM) in the simulation of band gap of PCs [32-35].

The purpose of the present paper is to explore the mechanism to tune the band gap in fluid and solid/fluid PCs using standard FEM. The local resonant elements, such as local resonant cavity in fluid PCs and local resonant intrusion in solid/fluid PCs, are introduced. First, we investigate the geometry of hole including convex and non-convex holes on the band gap of fluid PCs. Then, the effects of geometry parameters and material properties of solid scatterer on the band gap of solid/fluid PCs are further studied in this work. The thorough study in the analysis of band gap and sound transmission loss of fluid and solid/fluid PCs is able to provide a useful guideline to design PCs. The layout of the paper is structured as follows. In Section 2, the mathematical model for fluid and solid/fluid PCs 
using FEM is presented. The parameter study of band gap in fluid and solid/fluid PCs is analyzed in Section 3. The conclusions are made in Section 4.

\section{Stress wave model in fluid and solid/fluid PCs}

\subsection{Fluid PCs}

As shown in Fig.1, the hole is embedded into isotropic fluid. It is noted that only longitudinal wave exists in fluid PCs. The governing equation of longitudinal wave in fluid PCs is expressed as follows:

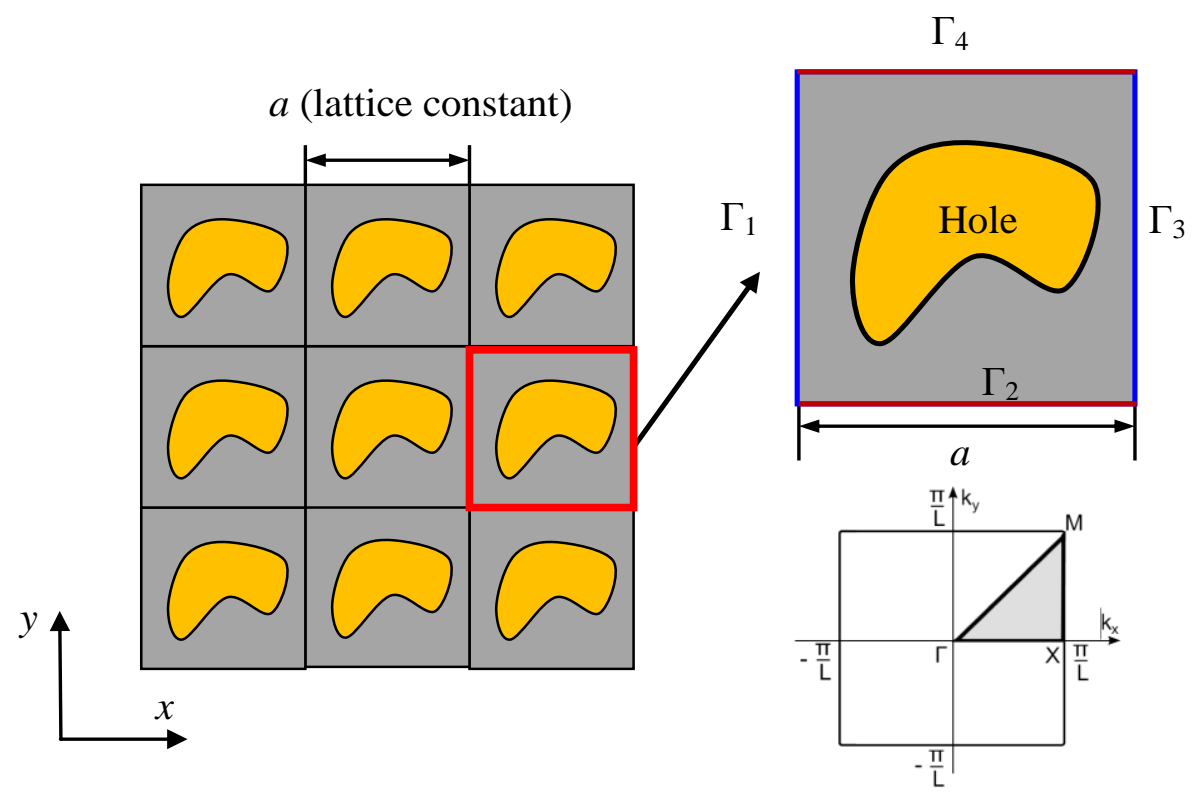

Figure 1: Fluid PCs with a hole

$$
\Delta p+\lambda^{2} p=0
$$

where $p$ is the pressure, $\Delta$ represents the Laplace operator, and $\lambda$ is the wavenumber which is defined as:

$$
\lambda=\frac{\omega}{c}
$$

where $\omega$ is the angular frequency, $c$ represents the speed of sound traveling in the fluid. 
According to the Bloch theorem, the following relation between the pressure $\mathbf{P}(\mathbf{r})$ for the nodes on the boundary of the unit cell is expressed as follows[36]:

$$
\mathbf{p}(\mathbf{r}+\mathbf{a})=e^{i(\mathbf{k} \cdot \mathbf{a})} \mathbf{p}(\mathbf{r})
$$

where $a$ is the lattice constant, $i=\sqrt{-1}, \mathbf{k}=\left(k_{x}, k_{y}\right)$ is the wave vector, and $\mathbf{p}_{\mathbf{k}}(\mathbf{r})$ is a periodic vector function with the same periodicity crystal lattice.

\subsection{Solid/fluid PCs with fluid-structural interaction}

In the solid/fluid PCs, the governing equation for the motion of the elastic wave in the solid domain with isotropic material is given by:

$$
\begin{aligned}
& \frac{\partial \sigma_{x x}}{\partial x}+\frac{\partial \sigma_{x y}}{\partial y}=\rho_{s} \frac{\partial^{2} u_{x}}{\partial t^{2}} \\
& \frac{\partial \sigma_{y y}}{\partial y}+\frac{\partial \sigma_{x y}}{\partial x}=\rho_{s} \frac{\partial^{2} u_{y}}{\partial t^{2}}
\end{aligned}
$$

where $u_{x}$ and $u_{y}$ are the induced displacements along $x$ and $y$ directions, respectively, $t$ is the time, and $\rho_{\mathrm{s}}$ is the density of solid.

The constitutive relationship for isotropic linear elasticity is expressed as follows:

$$
\boldsymbol{\sigma}=\mathbf{D \varepsilon}
$$

where $\mathbf{D}$ is a symmetric positive definite (SPD) matrix of material constant, which can be expressed as follows:

$$
\mathbf{D}=\left[\begin{array}{ccc}
\lambda+2 \mu & \lambda & 0 \\
\lambda & \lambda+2 \mu & 0 \\
0 & 0 & \mu
\end{array}\right] \quad \text { 2D plane strain }
$$

The Lame's constants $\mu$ and $\lambda$ are given as follows:

$$
\mu=\frac{E}{2(1+v)}, \lambda=\frac{v E}{(1+v)(1-2 v)}
$$


In the fluid part, the governing equation of longitudinal wave is the exactly the same as

\section{Section 2.1.}

Based on the continuity of displacement, the elastic and fluid particles move together in the normal direction of the interface as shown in Fig. 2.

$$
u_{s} \mathbf{n}=u_{f} \mathbf{n} \quad \text { on } \Gamma_{s f}
$$

where $\mathbf{n}$ is the normal vector, $u_{s}$ and $u_{f}$ denote the displacements of solid and fluid domains.

In addition, the structural stress and acoustic pressure in the interface can be written as follows:

$$
\begin{gathered}
\tau_{x}=\sigma_{x x} n_{x}+\sigma_{x y} n_{y}, \tau_{y}=\sigma_{y y} n_{x}+\sigma_{x y} n_{x} \\
\tau_{x}=p n_{x}, \tau_{y}=p n_{y}
\end{gathered}
$$

where $\tau$ represents the traction force.

The fluid force acting on the structure along the interface is given as follows:

$$
\mathbf{f}_{s}=\int_{\Gamma_{s f}} \mathbf{N}_{s}^{\mathrm{T}} \tau d \Gamma=\int_{\Gamma_{s f}} \mathbf{N}_{s}^{\mathrm{T}} \mathbf{n} p d \Gamma=\left(\int_{\Gamma_{s f}} \mathbf{N}_{s}^{\mathrm{T}} \mathbf{n} \mathbf{N}_{f} d \Gamma\right) \mathbf{p}
$$

where $\mathbf{N}$ is the shape function.

Similarly, the force from the structure exerting on the fluid is expressed:

$$
\mathbf{f}_{f}=-\rho_{f} \int_{\Gamma_{s f}} \mathbf{N}_{f}^{\mathrm{T}} \ddot{u}_{f} d \Gamma
$$

where $\rho_{f}$ is the density of fluid.

Define the spatial coupling matrix $\mathbf{S}$ as:

$$
\mathbf{S}=\int_{\Gamma_{s f}} \mathbf{N}_{s} \mathbf{n}_{f} \mathbf{N}_{f} \mathrm{~d} \Gamma
$$

The coupling forces in Eqs. (14) and (15) can be expressed as: 


$$
\mathbf{f}_{s}=\mathbf{S p}, \quad \mathbf{f}_{f}=-\rho \mathbf{S}^{\mathrm{T}} \ddot{\mathbf{u}}_{s}
$$

Based on Eqs. (15) and (16), the solid/fluid PCs with consideration of fluid-structural interaction can then be described by the following unsymmetrical equations:

$$
\left[\begin{array}{cc}
\mathbf{M}_{s} & 0 \\
\rho \mathbf{S}^{\mathrm{T}} & \mathbf{M}_{f}
\end{array}\right]\left[\begin{array}{c}
\ddot{\mathbf{u}}_{s} \\
\ddot{\mathbf{p}}
\end{array}\right]+\left[\begin{array}{cc}
\mathbf{K}_{s} & -\mathbf{S} \\
0 & \mathbf{K}_{f}
\end{array}\right]\left[\begin{array}{l}
\mathbf{u} \\
\mathbf{p}
\end{array}\right]=\left[\begin{array}{l}
\mathbf{f}_{s} \\
\mathbf{f}_{f}
\end{array}\right]
$$

Assume that the displacement and pressure are time harmonic, the force frequency response analysis equation for solid/fluid PCs can be derived:

$$
\left[\begin{array}{cc}
\mathbf{K}_{s}-\omega^{2} \mathbf{M}_{s} & -\mathbf{S} \\
\rho_{f} \omega^{2} \mathbf{S}^{\mathrm{T}} & \mathbf{K}_{f}-\omega^{2} \mathbf{M}_{f}
\end{array}\right]\left[\begin{array}{l}
\mathbf{u} \\
\mathbf{p}
\end{array}\right]=\left[\begin{array}{c}
\mathbf{f}_{s} \\
\mathbf{f}_{f}
\end{array}\right]
$$

By mean of FEM, the mass and stiffness matrices in Eq. (18) are expressed as follows:

$$
\begin{array}{lr}
\mathbf{M}_{s}=\rho_{s} \int_{\Omega} \mathbf{N}_{s}^{\mathrm{T}} \mathbf{N}_{s} \mathrm{~d} \Omega & \text { The mass matrix in the solid domain } \\
\mathbf{K}_{s}=\int_{\Omega} \nabla \mathbf{N}_{s}^{\mathrm{T}} \mathbf{D}_{s} \nabla \mathbf{N d} \Omega & \text { The stiffness matrix in the solid domain } \\
\mathbf{M}_{f}=\rho_{f} \int_{\Omega} \mathbf{N}_{f}^{\mathrm{T}} \mathbf{N}_{f} \mathrm{~d} \Omega & \text { The mass matrix in the fluid domain } \\
\mathbf{K}_{f}=\int_{\Omega} \nabla \mathbf{N}_{f}^{\mathrm{T}} \nabla \mathbf{N}_{f} \mathrm{~d} \Omega & \text { The stiffness matrix in the fluid domain }
\end{array}
$$

In the solid/fluid PCs, only the acoustic wave is applied to the periodic boundary. For a square lattice, the pressure should satisfy the following equation [24, 27, 37]:

$$
\mathbf{p}\left(\mathbf{x}_{\Gamma_{1}}\right)=\mathbf{p}\left(\mathbf{x}_{\Gamma 3}\right) e^{-i k_{x} a}, \mathbf{p}\left(\mathbf{x}_{\Gamma_{2}}\right)=\mathbf{p}\left(\mathbf{x}_{\Gamma_{4}}\right) e^{-i k_{y} a}
$$

In the simulation of wave propagation, the dispersion error is a critical issue from numerical perspective. The latest methods, such as meshfree methods [38], smoothed finite element method (S-FEM) [26, 39-44] and mass-redistributed finite element method (MRFEM) $[32,45,46]$ might be a better choices. This is because these methods can be more accurate in natural frequency prediction due to the softening effects. As the main objective 
of this work is to reveal the mechanism of band gap in fluid and solid/fluid PCs, the comparison of accuracy using FEM and other types of meshfree method is ignored in this paper.

\section{Results and Discussions}

\subsection{Fluid PCs}

In this section, we first discuss the fluid PCs with different types of hole using commercial software COMSOL. As shown in Fig. 2, the properties of water are taken as follows: the density $\rho=1000 \mathrm{~kg} / \mathrm{m}^{3}$, and the speed of sound in the water is $c=1500 \mathrm{~m} / \mathrm{s}$. In this study, three typical holes including square, circular and "+” geometry are employed.
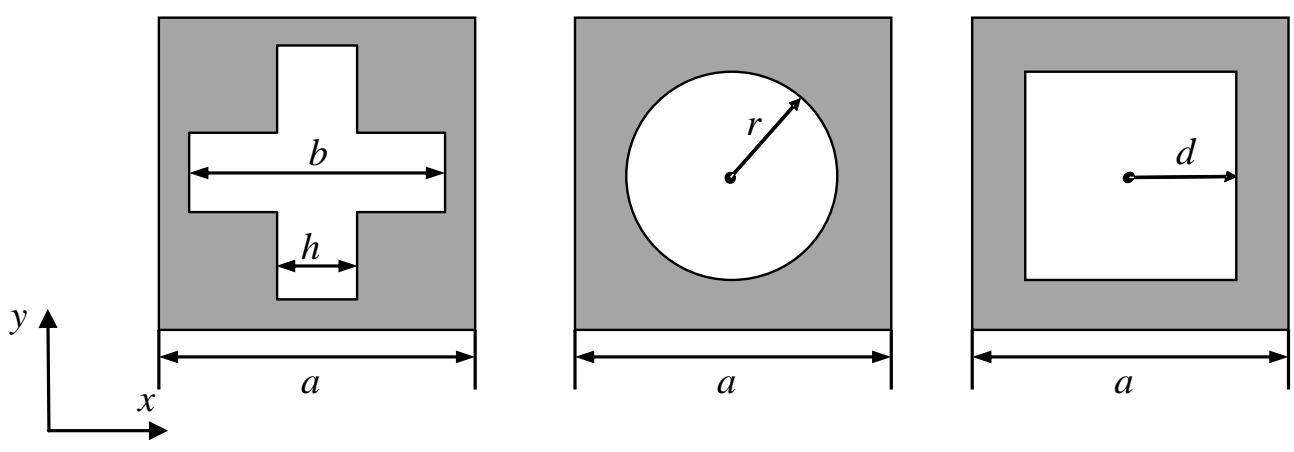

Figure 2: Fluid PCs with different geometry of hole $(a=0.15 \mathrm{~m})$

In order to study the geometry effect, the maximum size for these three different holes is identical. As shown in Fig. 3, the results of band gap for fluid PCs with different types of hole are presented. It is seen that the fluid PCs with "+" and circular holes have multiple band gaps. However, the fluid PCs with "+" hole is able to produce a wider band gap which is more effective to stop / block the wave propagation. Due to the geometry effect, it is noticed that there is no band gap in the fluid PCs with square hole. As illustrated in Fig. 3, the normalized frequency is almost the same order of size of lattice constant in the fluid PCs with "+" and "circular" holes. This verifies that the appearance of band gap in fluid 
PCs with "+" and circular holes is due to the mechanism of Bragg scattering. Compared with solid PCs, it is noted that only longitudinal wave propagates within the fluid PCs. According to the computed results, it is clearly seen that the geometry of hole plays a crucial role to control/manipulate the propagation of longitudinal wave in the design of fluid PCs. The theoretical analysis presented in this work is quite useful to design fluid sensor and other related area such as biomedical engineering.

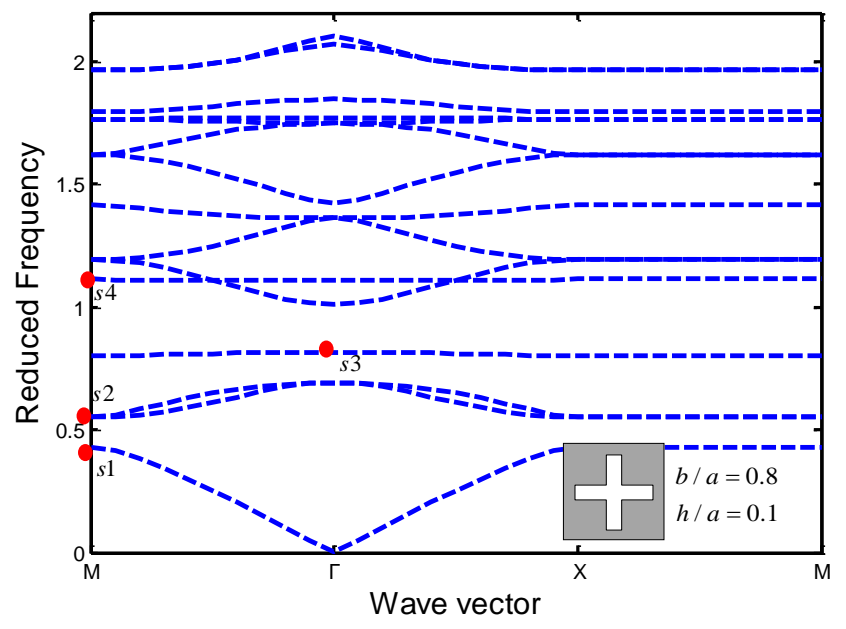

(a) "+" hole

1

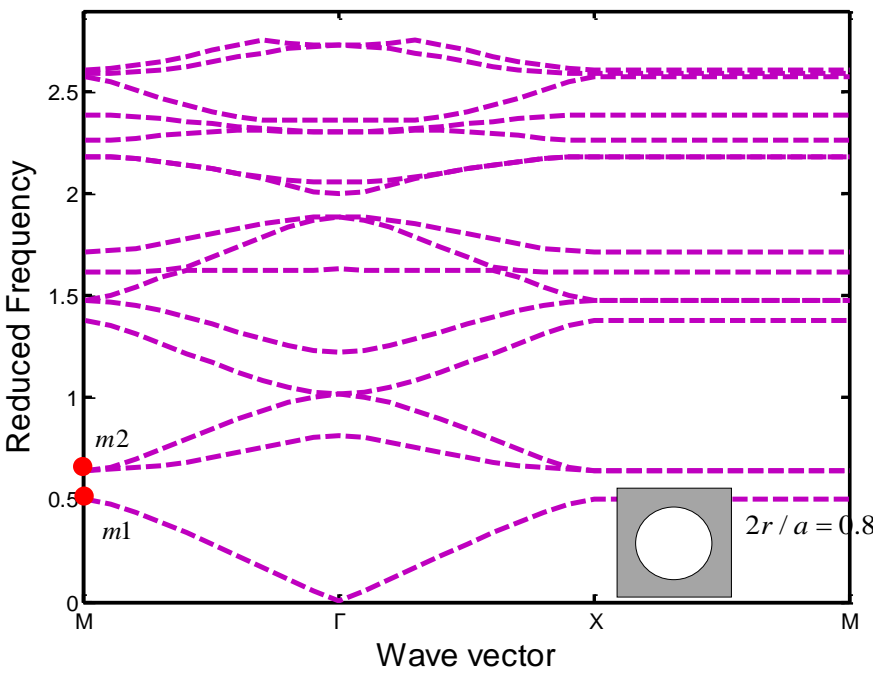

(b) Circular hole 


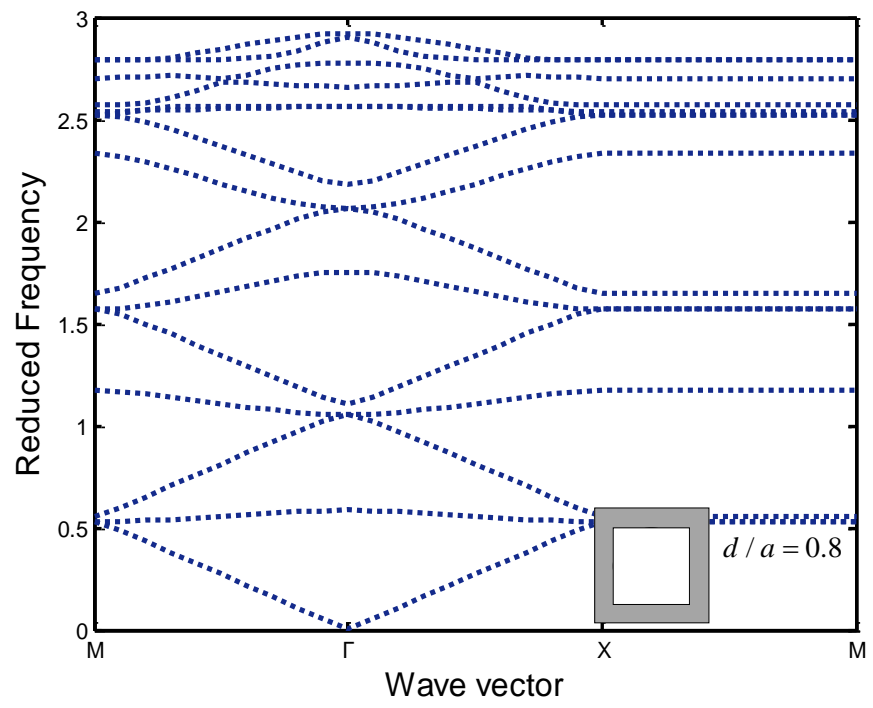

(c) Square hole

Figure 3: Band gap for different types of hole

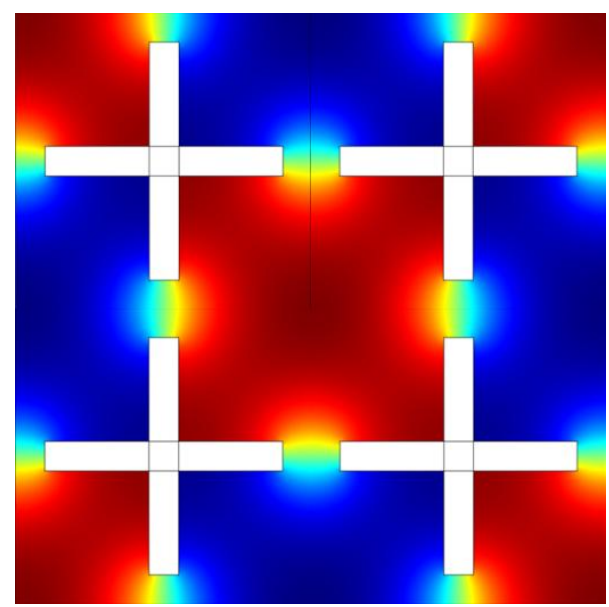

(a) "+" hole at $S 1$ point

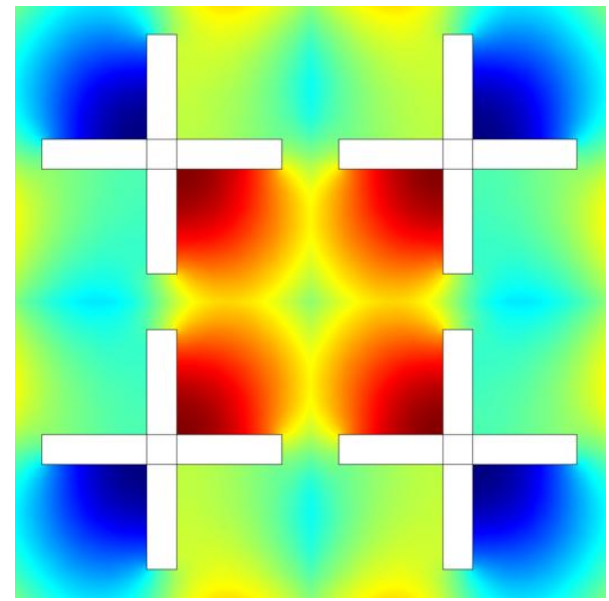

(c) "+" hole at $S 3$ point

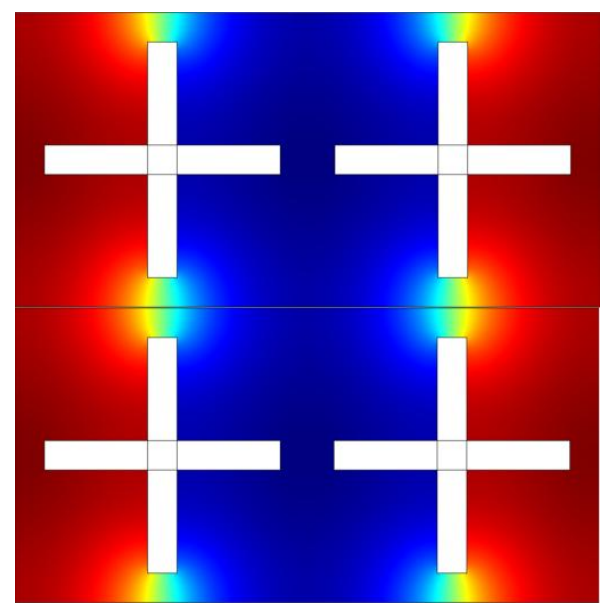

(b) "+" hole at $S 2$ point

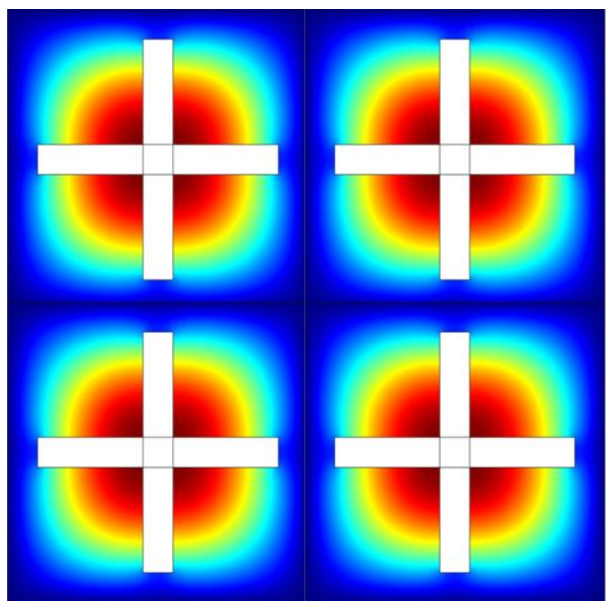

(d) "+" hole at $S 4$ point 


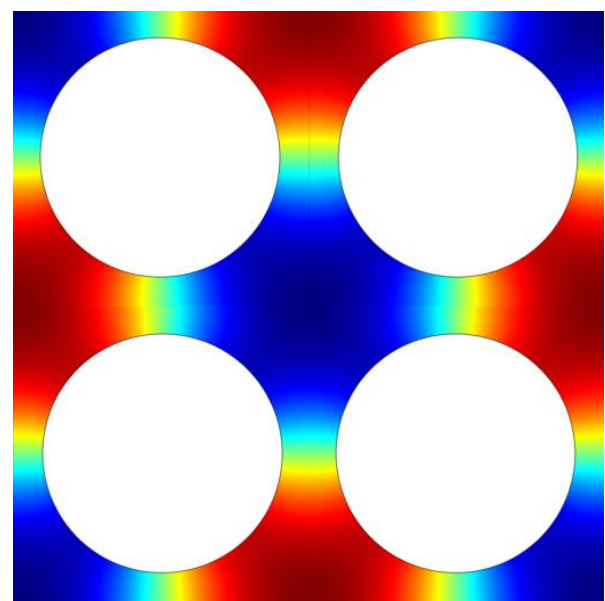

(e)Circle hole at $m 1$ point

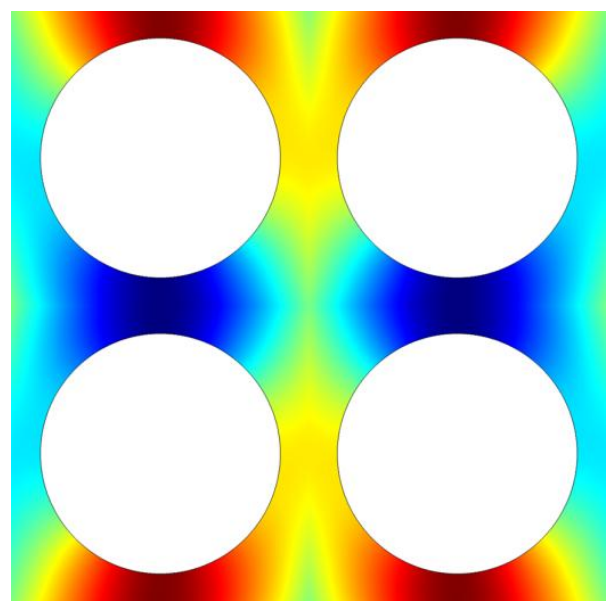

(f) Circle hole at $m 2$ point

Figure 4: Comparison of vibration mode shape

Next, the mode shapes of the fluid PCs with "+" and circular holes are shown in Fig. 4. As outlined in Fig. 4(a), the mode shapes at the edge of largest band gap (at point $s 1$ ) for fluid PCs with "+" hole and the mode shape at point $s 1$ with circle hole are similar to Helmholtz Resonator. In addition, the second mode shape for both fluid PCs with "+"and circle holes are the half of wave length. It is worth noting that the third and the fourth mode shapes for fluid PCs with "+" hole are due to the local resonant effect.

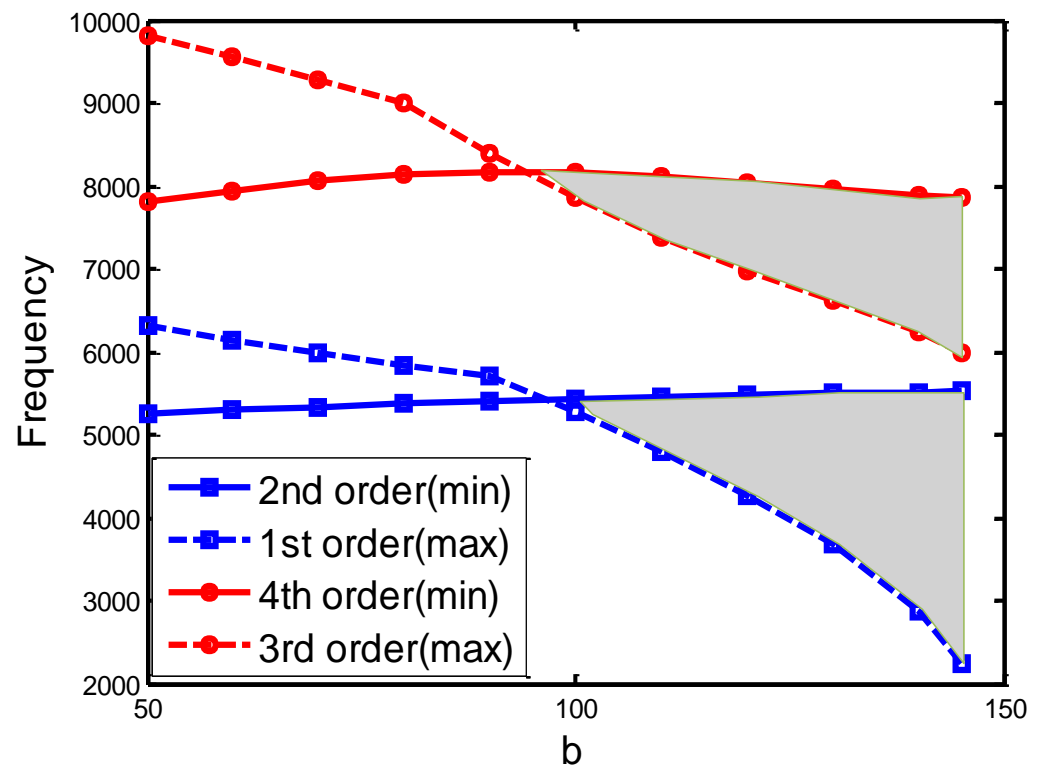

(a) Effect of $b$ on the band gap $(h=15 \mathrm{~mm})$ 


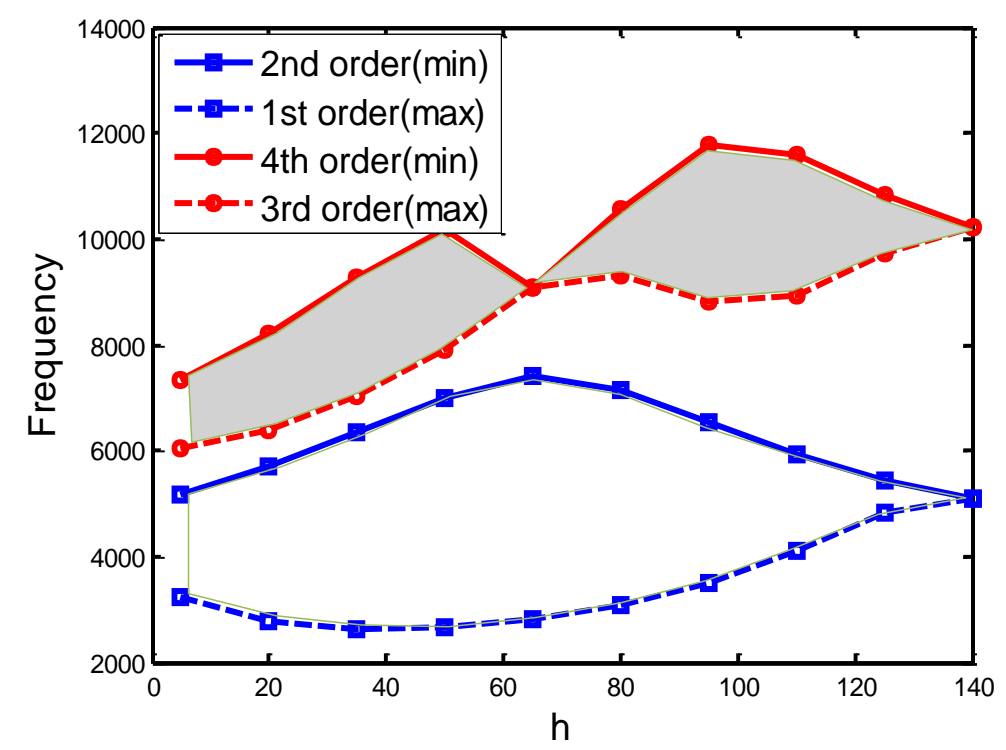

(b) Effect of $h$ on the band gap $(b=140 \mathrm{~mm})$

Figure 5: Effects of the geometry size on the band gap in fluid PCs

Then, we study the mechanism why the fluid PCs with "+" hole is able to provide multiple band gaps. Fig. 5 shows the effect of parameters $b$ and $h$ on the band gap in the fluid PCs with "+" hole. As outlined in Fig. 5(a), it is observed that the second and fourth frequencies remain nearly unchanged when the parameter $b$ varies from $50 \mathrm{~mm}$ to $140 \mathrm{~mm}$. This is because that the eigenfrequencies are almost the same order of size of lattice constant, which is related to the size of PCs. It is also observed that the first eigenfrequency becomes smaller with the increase of $b$, which is the result from the characteristic of local Helmholtz Resonator eigenfrequency computed in Eq. (24) and Fig. 6. Based on Eq. (24), it is seen that as $l_{c}$ ( $h$ is analogous to $l_{c}$ in the fluid PCs with "+" hole ) and the volume $V$ keep constant, $S_{c}\left((a-b)\right.$ is analogous to $S_{c}$ in the fluid PCs with "+" hole ) becomes smaller, leading to the decrease of the first eigenfrequency. Therefore, a much larger band gap width is obtained by introducing the local resonant cavity in the fluid PCs. 
Based on the above analysis, we can make a mini conclusion here: the adjustment of $b$ leads to the change of eigenfrequencies of local resonant cavity, which is able to effectively control the width of band gap. This rule can also be applied to Fig. 5(b) when it comes to the change of the parameter of $h$. Due to the local resonant elements in the cavity, the generation of the wide low-frequency band gaps is obtained for the fluid PCs with "+" hole.

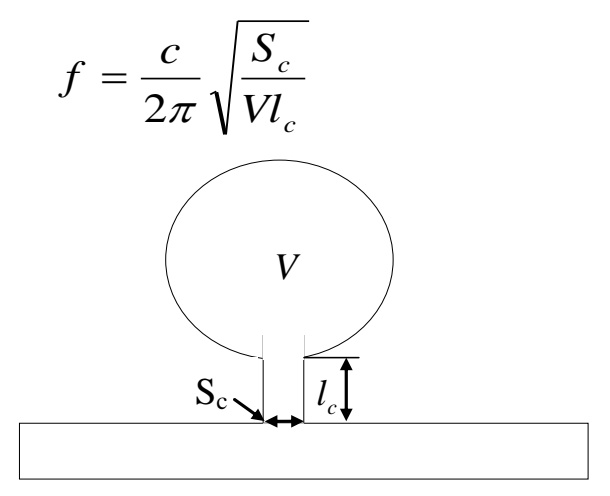

Figure: 6 Model of Helmholtz Resonator

\subsection{Solid/fluid PCs}

In Section 3.1, it is seen that fluid PCs with "+" hole can show better performance in terms of wider multi band gaps compared with circular hole due to geometry effect. In this subsection, the local resonant intrusion is introduced (The "+", square and circle holes in the fluid PCs are replaced by solid scatterer), which is terms as solid/fluid PCs. In the computation of solid/fluid PCs, the effect of fluid structure interaction on the band gap is considered. Two kinds of solid i.e. aluminum (Young's modules $E_{\mathrm{Al}}=71 \mathrm{GPa}$, Poisson ratio $v_{\mathrm{Al}}=0.3$, and density $\rho_{\mathrm{Al}}=2700 \mathrm{~kg} / \mathrm{m}^{3}$ ) and epoxy (Young's modules $E_{\mathrm{Ep}}=3.6 \mathrm{GPa}$, Poisson ratio $v_{\mathrm{Ep}}=0.37$, and density $\rho_{\mathrm{Ep}}=1180 \mathrm{~kg} / \mathrm{m}^{3}$ ) are immersed in the water (sound speed $c=1500 \mathrm{~m} / \mathrm{s}$, and density $\rho=1000 \mathrm{~kg} / \mathrm{m}^{3}$ ) and mercury (sound speed $c=1451 \mathrm{~m} / \mathrm{s}$, and density $\left.\rho=13600 \mathrm{~kg} / \mathrm{m}^{3}\right)$, respectively. 
The first fifteen eigenfrequencies for both aluminum and epoxy immersed in water and mercury are presented in Fig. 7. In the fluid PCs with "+" hole without consideration of fluid-structural interaction, we can obtain the band gap in the high frequency region based on Bragg scattering mechanism. However, the frequency in the region of band gap appears in solid/fluid PCs is much lower with taking fluid-structural interaction into consideration, i.e water PCs with aluminum inclusion or mercury PCs with aluminum inclusion. The fluid PCs with "+" hole can be considered as the solid with extremely high stiffness immersed in the water. Therefore, the following mini-conclusion can be made: the selection of solid scatterer with different material property is crucial to create the band gap in solid/fluid PCs. In addition, as indicated in Fig.7, it is noticed that the frequency of band gap is strongly dependent on the solid property. The frequency of band gap can be lower with introducing softer solid scatterer. In contrast, the properties of fluid play a very minor effect on the frequency of band gap.

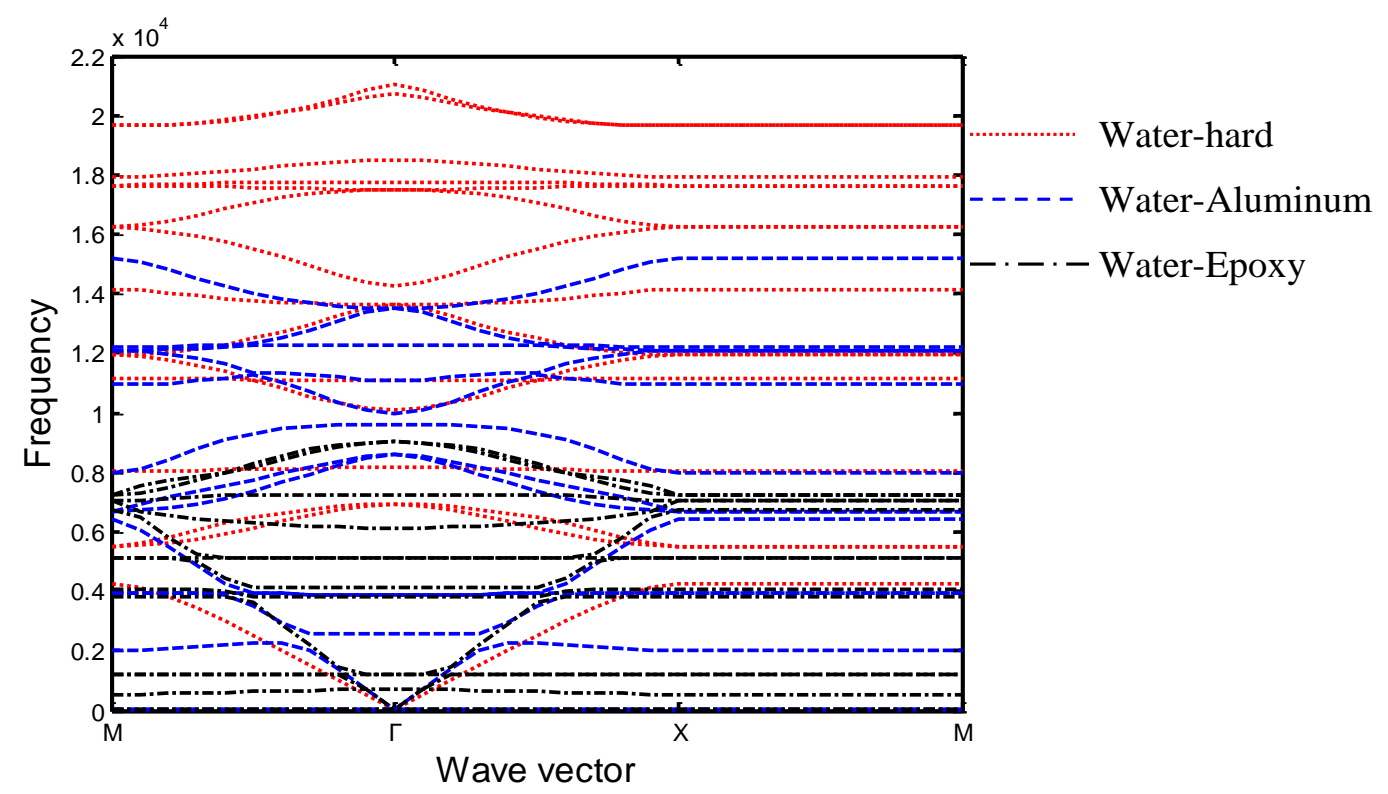




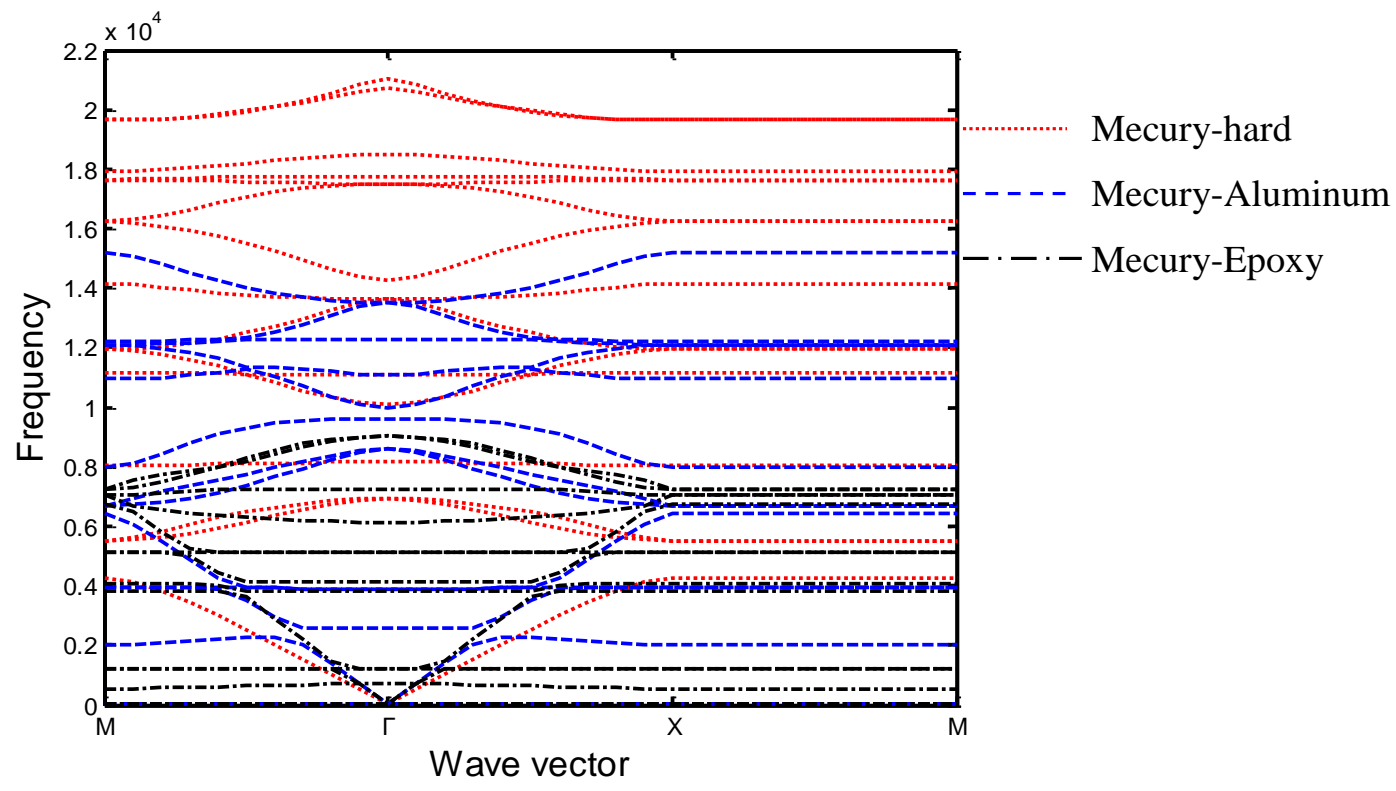

Figure 7: Band gap analysis (red and blue colour stands for fluid and solid/fluid PCs)

The control of low frequency wave is very important in the practical applications. In order to obtain lower frequency band gap, we then consider a relatively soft material rubber as solid scatterer instead of aluminum, which is immersed in the water, and the band gaps for different geometry of solid in solid/fluid PCs are illustrated in Fig. 8. In this case, the materials properties for the rubber are taken as follows: Young's modules $E=11.75 \times 10^{5}$ Pa, Poisson's ratio $v=0.35$ and density $\rho=1300 \mathrm{~kg} / \mathrm{m}^{3}$.

As outlined in Fig. 8, it is interesting and worthy noted that the band gaps due to locally resonant appear in all solid/fluid PCs with different geometries of solid scatterer. More importantly, the low frequency band gap can be obtained in solid/fluid PCs compared with high frequency band gap of fluid PCs caused by Bragg scattering. Obviously, the solid/fluid PCs with consideration of fluid-structural interaction can give local resonant modes, which is lower order of size of lattice constant. The mechanism of local resonant mode in solid/fluid PCs can be used to control the propagation of longitudinal wave in fluid at low 
frequency range or design the fluid sensor. Furthermore, it is clearly seen that the geometry of rubber plays a crucial role to control/manipulate the propagation of stress wave in the design of solid/fluid PCs according to the computed results.

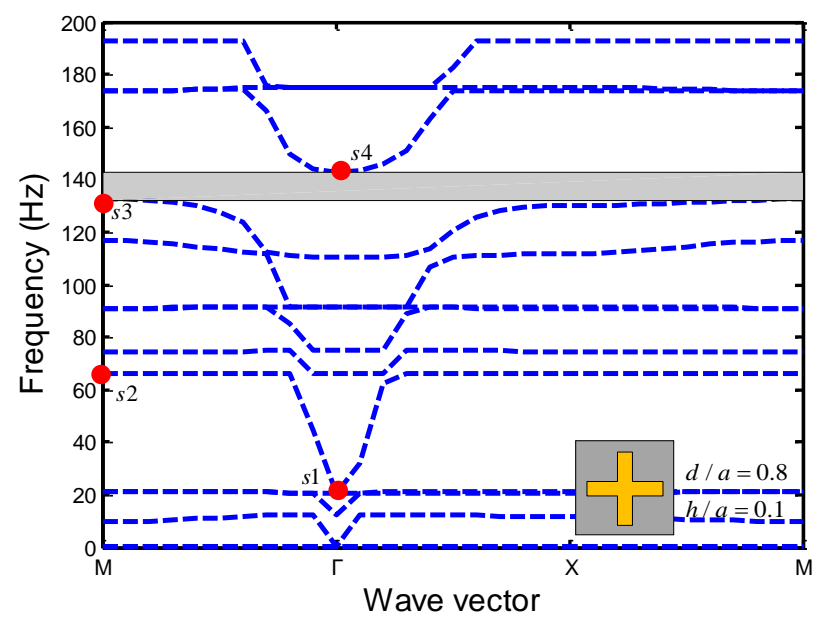

(a) "+" solid

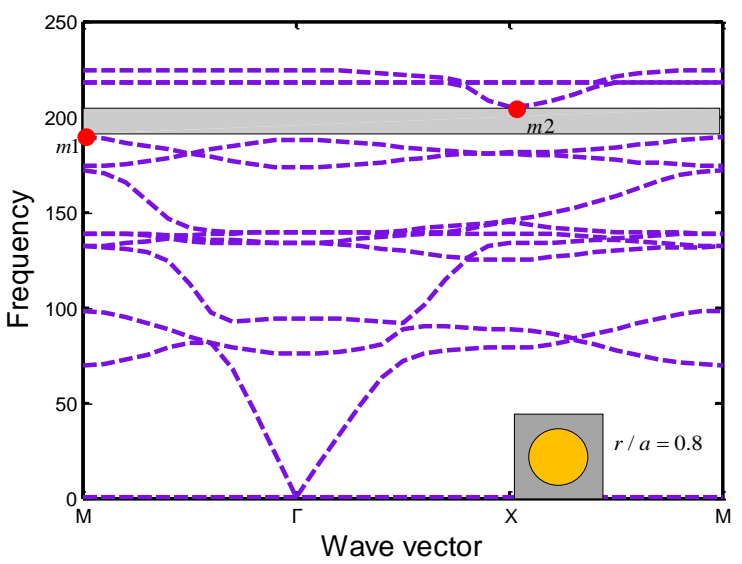

(b) Circular solid

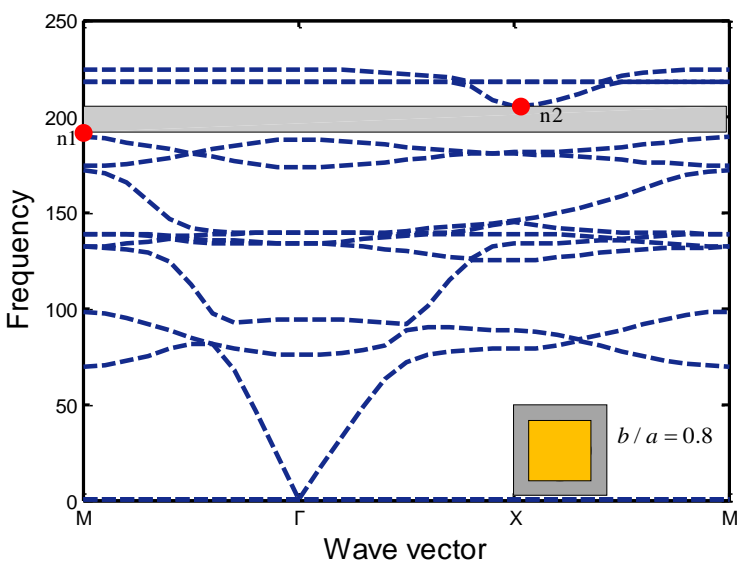

(c) Square solid

Figure 8: Band gap for different types of solid/fluid PCs 
In order to further explore the mechanism of band gap in solid/fluid PCs with "+" geometry, the mode shapes of $s 3$ and $s 4$ for solid and fluid domains are plotted in Fig. 9. It is found that the vibration modes are strongly related to compression and tension status.

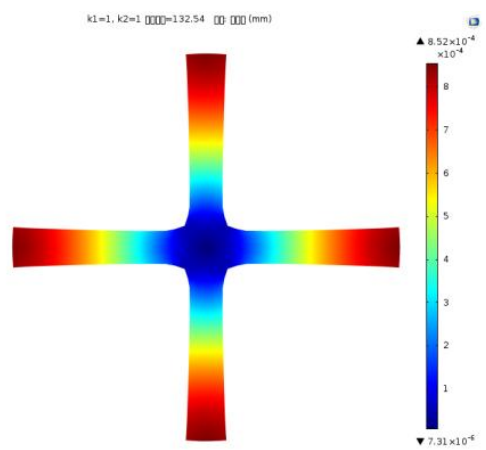

(a) S3 (solid)

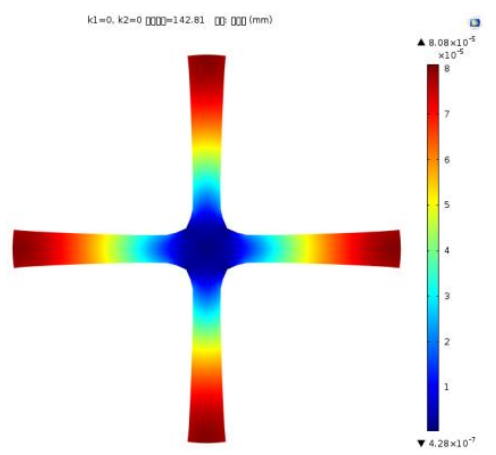

(c) S4 (solid)

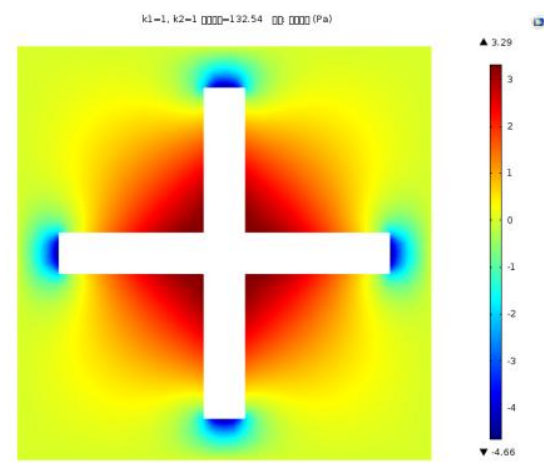

(b) S3 (fluid)

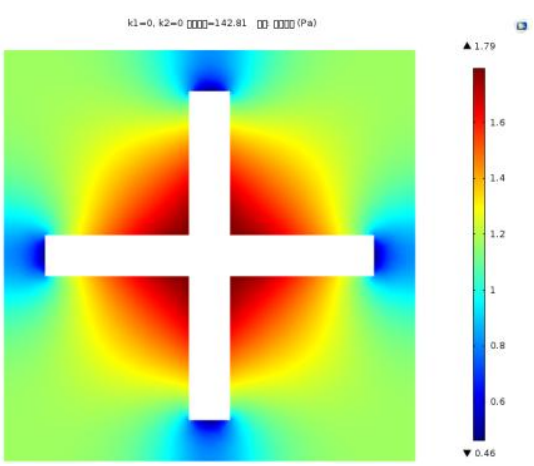

(d) S4 (fluid)

Figure 9: Vibration modes for solid and fluid domains

The about results as outlined in Fig. 9 indicate that the vibration mode shape can be modeled as one-dimensional tension or compression of the rectangular strip. Thus, the frequency can be obtained using the following formula:

$$
\omega=\frac{\left(n-\frac{1}{2}\right) \pi}{l} \sqrt{\frac{E}{\rho}}, \quad n=1,2,3, \ldots
$$

For the first mode shape, we can have the following frequency: 


$$
\begin{aligned}
& \omega=\frac{\pi}{2 l} \sqrt{\frac{E}{\rho}}=\frac{3.142}{0.12} \sqrt{\frac{11.75 e 5}{1300}}=787.0746 \\
& f=\omega / 2 \pi=125.2668 \mathrm{~Hz}
\end{aligned}
$$

The frequency obtained from Eq. (26) is quite close to the eigenfrequency as outlined in Fig. 9(a). However, the above analysis ignores the effect of two-dimensional deformation. In general, the frequency in the fluid-structural interaction model is higher than that in the pure strip model. Next, we study different parameters on the band gap of solid/fluid PCs with “+” scatterer.

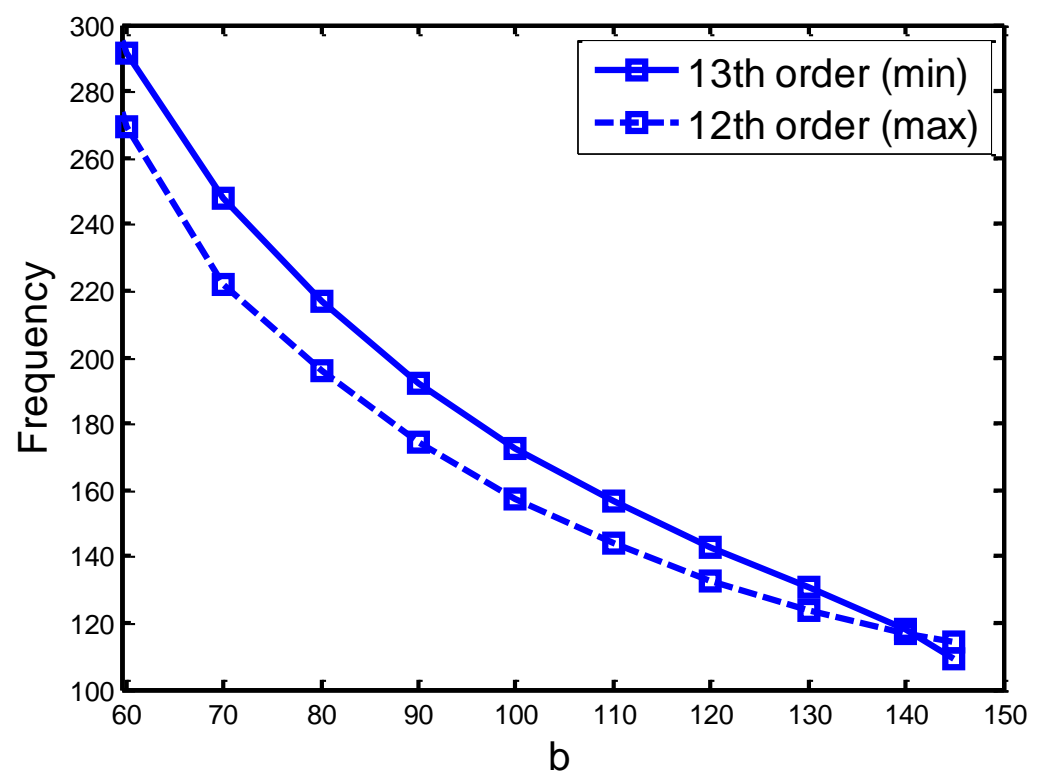

Figure 10: Effect of $b$ in solid/fluid PCs with "+" scatterer

Figure 10 analyzes the effect of $b$ on the band gap of solid/fluid PCs with "+" scatterer. It is easily noticed that the eigenfrequencies of $12^{\text {th }}$ and $13^{\text {th }}$ order become smaller with the increase of $b$, which agrees very well with the theoretical results in Eq.(25). It also observed that the parameter $b$ has a minor influence on the band gap of solid/fluid PCs. On the contrary, the parameter $b$ has a significant effect on the band gap of fluid PCs with "+" hole as outlined in Fig. 6(a). This is because the mechanism of the band gap for fluid PCs and solid/fluid PCs is totally different, i.e. the band gap for fluid PCs is based on 
the local resonant cavity, while the band gap for solid/fluid PCs is based on the local resonant inclusion. Consequently, we study the effect of $h$ on the band gap as outlined in Fig. 11. As $h \leq 50$, the vibration shape of solid scatterer depends on the tension and compression state, and the width of band gap increases based on the increase of the tension and compression effect. As $h \geq 60$, the vibration mode shape changes significantly, which leads to another mode of band gap. It is concluded that the mode shape of tension and compression effects play an important part in the width of band gap.

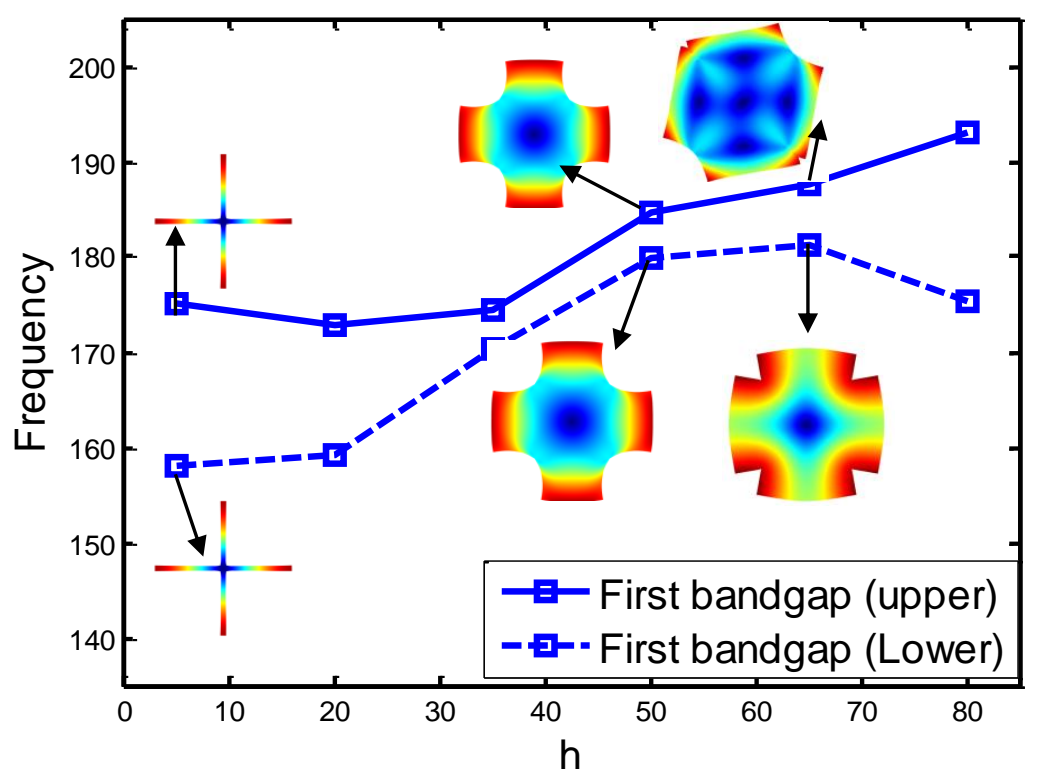

Figure 11: Effect of $h$ in solid/fluid PCs with “+” scatterer $(b=100 \mathrm{~mm})$

In order to further discuss the effect of mode shape of resonator, we investigate the effect of Poisson's ratio of rubber, which plays an important part on the band gap due to the effect of tension and compression. Because of the manufacturing, the rubber can be compressible and incompressible. As shown in Fig. 12, the effect of Poisson's ratio of rubber on the band gap of solid/fluid PCs is presented. It is seen that the Poisson's ratio of rubber has a significant effect on the band gap of solid/ fluid PCs with "+" scatterer. With increase of the Poisson's ratio of rubber, the band gap is becoming narrower and narrower. 
When the Poisson's ratio is close to 0.5 , the band gap almost disappears, which is also in accordance with the local resonant mode of tension and compression.

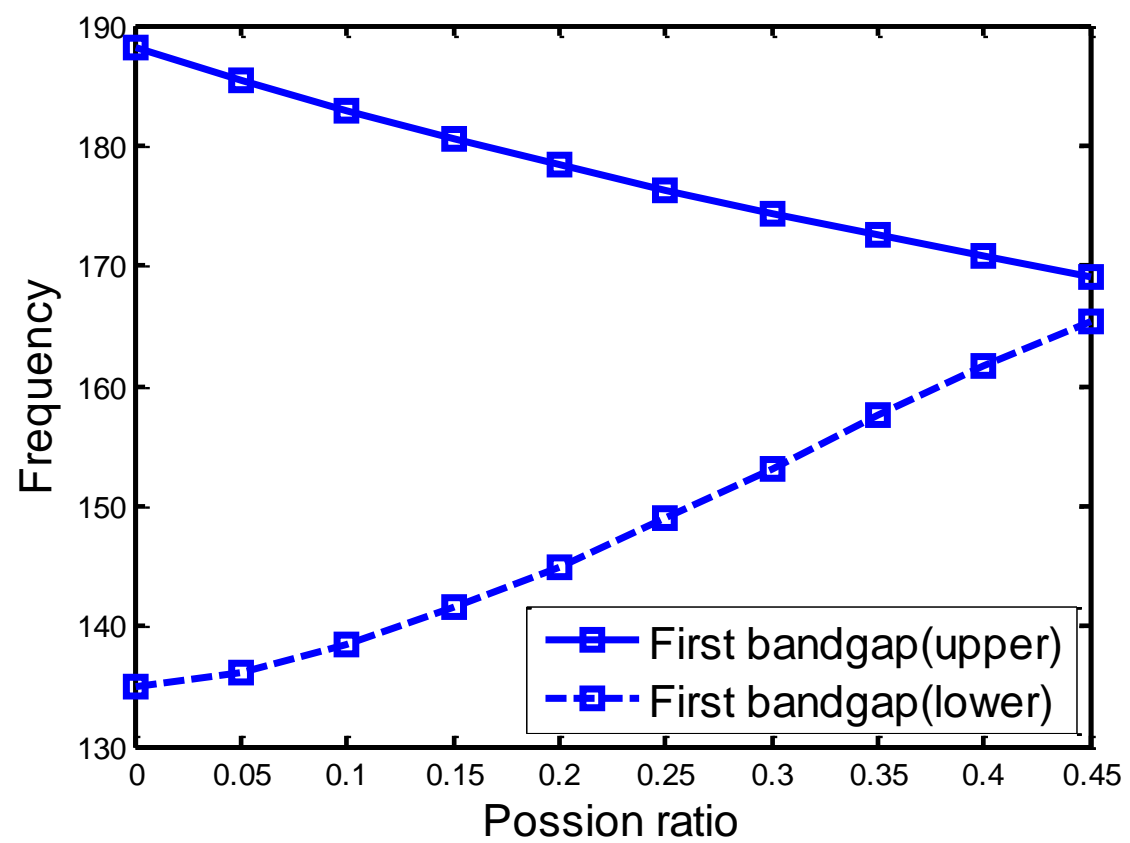

Figure 12: The effect of Poisson's ratio on the band gap

\subsection{Sound transmission loss in PCs}

The study of sound transmission loss (STL) through the engineering structure such as beams, plates and membranes is of great importance. In the past, substantial research has been paid to the STL in solid-solid PCs. However, very little research has focused on the STL in the fluid PCs. In particular, to the best of our knowledge, the effect of STL on the solid/fluid PCs is seldom reported in the literature. The objective of this section is to investigate the STL of solid/fluid and fluid PCs consisting of different geometry. As shown in Fig. 13, the solid scatterer with different geometry is immersed into the water. In this example, the properties of solid scatterer are taken as follows: Young's modules $E=$ $11.75 \times 10^{5} \mathrm{~Pa}$, Poisson's ratio $v=0.35$ and density $\rho=1300 \mathrm{~kg} / \mathrm{m}^{3}$. In the case without consideration of fluid-structural interaction, the solid is assumed as rigid material. The right hand side is a perfectly matched layer (PML) in the study of STL. 


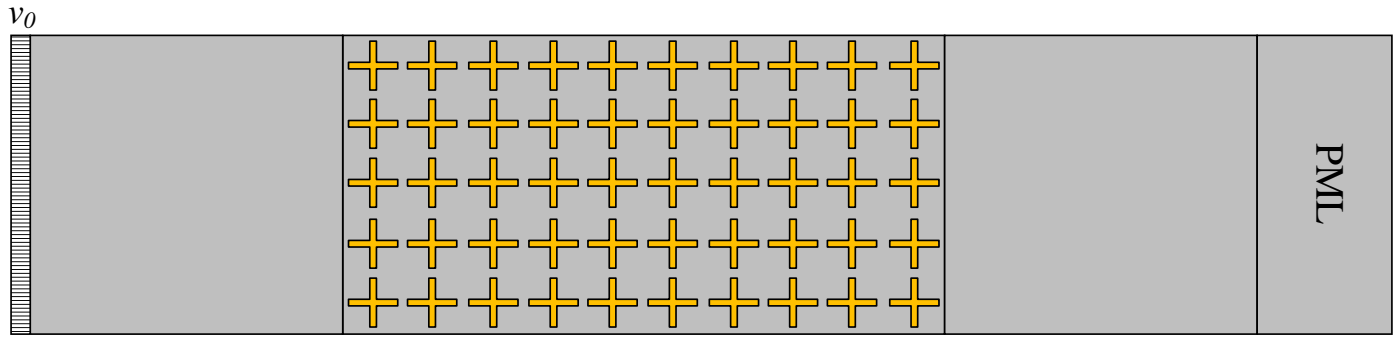

(a) “+” scatterer

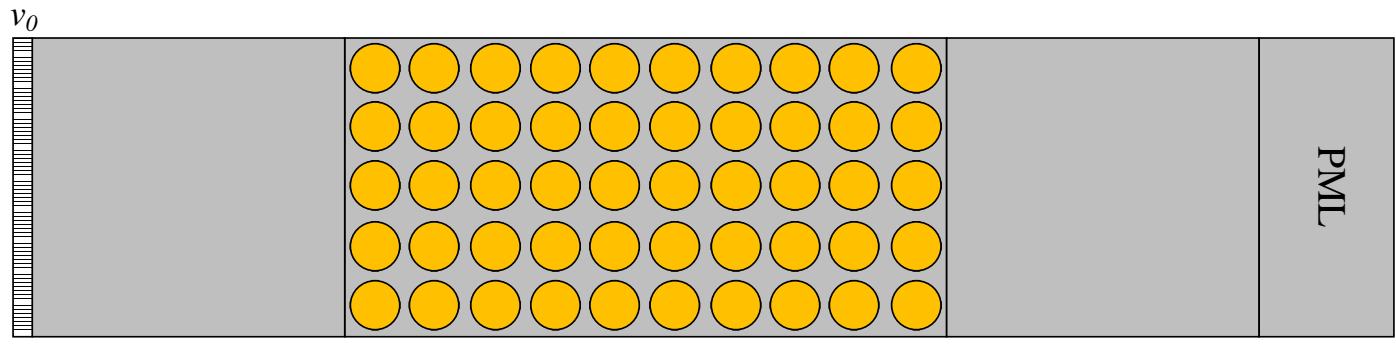

(b) "circular" scatterer

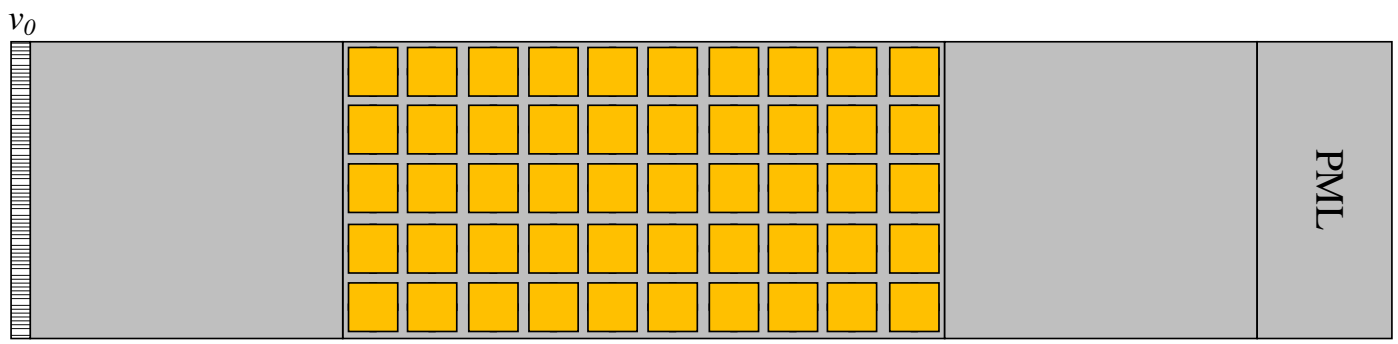

(c) "square" scatterer

Figure 13: The model of STL

The sound transmission loss is defined as:

$$
\mathrm{T} L=20 \log \left(\left|p_{\text {incident }} / p_{\text {transmitted }}\right|\right)
$$

where $\mid p_{\text {incident }}$ is the incident wave, and $p_{\text {transmitted }}$ is the transmitted wave. 


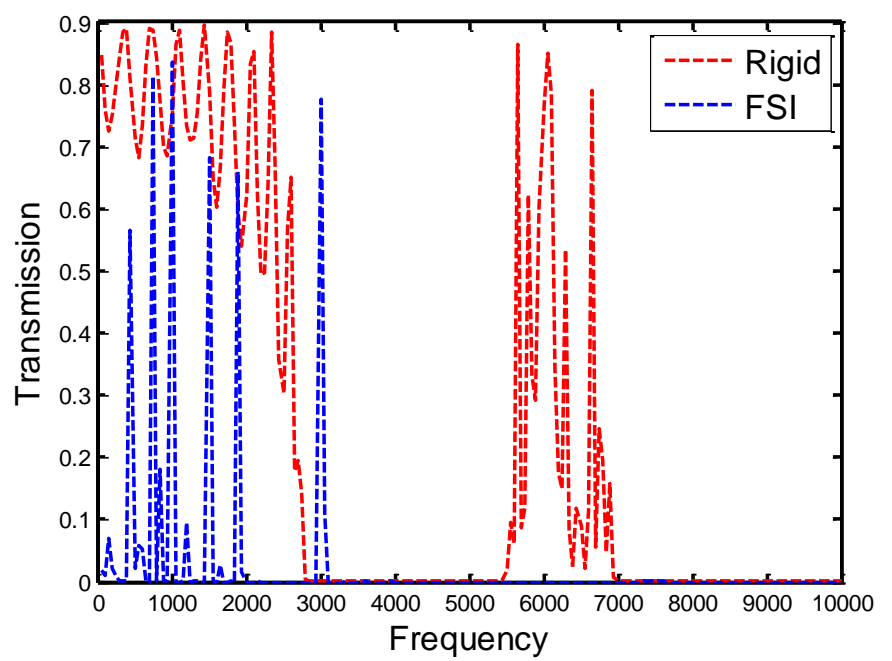

(a) +

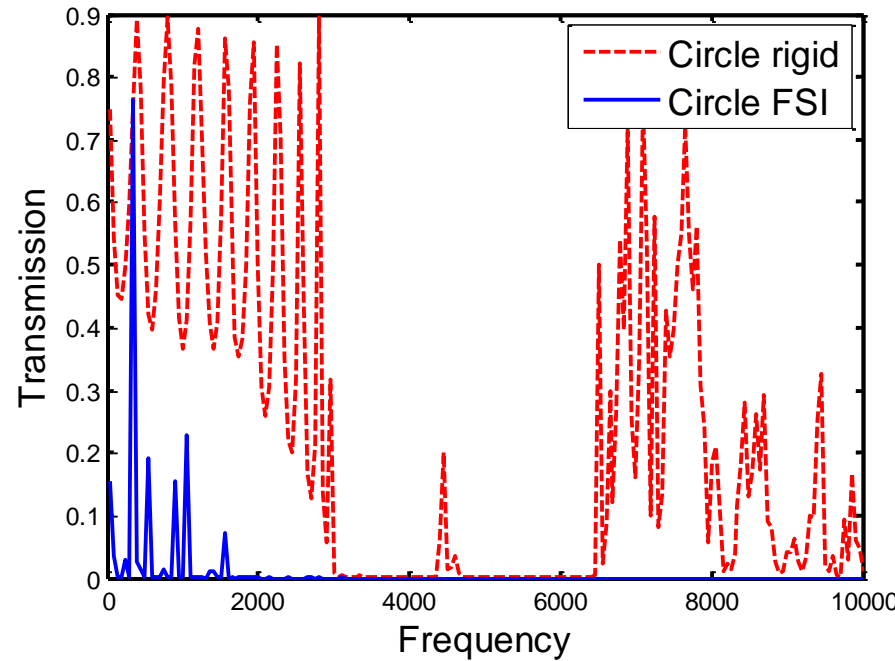

(b) Circle

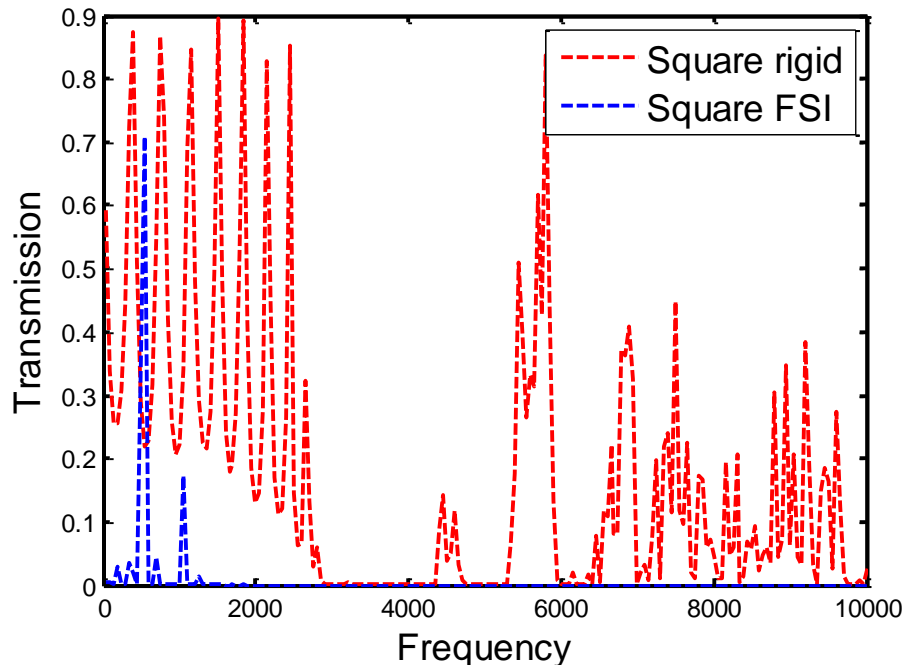

(c) Square

Figure 14: Comparison of sound transmission loss 
Figure 14 illustrates the results of STL with different geometries of scatterer. It is clearly seen that the coupling effect between fluid and solid can block the propagation of sound wave effectively especially in very low frequency range by introducing the local resonant inclusion, leading to the transmission loss reduced significantly compared with fluid PCs without consideration of fluid-structural interaction. The important finding discovered in this work provides a useful guideline to design novel materials and structures in order to improve the performance of STL.

\section{Conclusion}

In this work, three types holes including square, circular and "+" geometry are considered to study the band gap and sound transmission loss of fluid and solid/fluid PCs. The effects of the geometry parameters of the holes on the band gaps are discussed in detail. The mechanism of the generation of the lowest band gap is analyzed by studying the vibration modes of the band gap edges. From the calculated results and discussions, the following conclusions can be made:

(1) The characteristic of band gap in the fluid PCs strongly depends on the size and geometry of the hole, and fluid PCs with "+" hole can produce wider multiple band gaps in contrast with circular and square holes.

(2) The appearance of band gap in the solid/fluid PCs with taking fluid-structural interaction into account is due to locally resonance, which is able to create the very low frequency band gap.

(3) The Young's modulus and Poisson's ratio of solid are essential to control the band gap of solid/fluid PCs. In addition, the size and geometry of solid scatterer play an important role to determine the band gap of solid/fluid PCs. 
(4) The variance of band gap in solid/fluid PCs for different properties of fluid is minor.

(5) The performance of sound transmission loss in solid/fluid PCs with consideration of fluid-structural interaction is better than fluid PCs, which can be used to control the very low and high frequency wave effectively.

\section{Acknowledgements}

The project is supported by the Science Fund of State Key Laboratory of Advanced Design and Manufacturing for Vehicle Body No. 31615002. The authors wish to thank and Research Project of State Key Laboratory of Mechanical Systems and Vibration MSV201711 and Research Project of State Key Laboratory of Structural Analysis for Industrial Equipment (Grant No. GZ 1609) for the support. 


\section{Reference}

[1] Hussein MI, Frazier MJ. Band structure of phononic crystals with general damping. Journal of Applied Physics. 2010;108:093506.

[2] Li FL, Wang YS, Zhang CZ. Bandgap calculation of two-dimensional mixed solid-fluid phononic crystals by Dirichlet-to-Neumann maps. Physica Scripta. 2011;84.

[3] Lin S-CS, Huang TJ. Tunable phononic crystals with anisotropic inclusions. Physical Review B. 2011;83:174303.

[4] Hussein MI, Leamy MJ, Ruzzene M. Dynamics of Phononic Materials and Structures: Historical Origins, Recent Progress, and Future Outlook. Applied Mechanics Reviews. 2014;66.

[5] Wang Y-Z, Li F-M, Wang Y-S. Influences of active control on elastic wave propagation in a weakly nonlinear phononic crystal with a monoatomic lattice chain. International Journal of Mechanical Sciences. 2016;106:357-62.

[6] He ZC, Xiao X, Li E. Design for structural vibration suppression in laminate acoustic metamaterials. Composites Part B: Engineering. 2017;131:237-52.

[7] Viktor GV. The electrodynamics of substances with simultaneously negative values of $\epsilon$ and $\mu$. Soviet Physics Uspekhi. 1968;10:509.

[8] Zheng LY, Wu Y, Ni X, Chen ZG, Lu MH, Chen YF. Acoustic cloaking by a near-zeroindex phononic crystal. Applied Physics Letters. 2014;104.

[9] Ke MZ, Zubtsov M, Lucklum R. Sub-wavelength phononic crystal liquid sensor. Journal of Applied Physics. 2011;110.

[10] Sukhovich A, Merheb B, Muralidharan K, Vasseur JO, Pennec Y, Deymier PA, et al. Experimental and Theoretical Evidence for Subwavelength Imaging in Phononic Crystals. Physical Review Letters. 2009;102.

[11] Lv HY, Tian XY, Wang MY, Li DC. Vibration energy harvesting using a phononic crystal with point defect states. Applied Physics Letters. 2013;102.

[12] Shen L, Wu JH, Zhang SW, Liu ZY, Li J. Low-frequency vibration energy harvesting using a locally resonant phononic crystal plate with spiral beams. Modern Physics Letters B. $2015 ; 29$.

[13] Lucklum R, Li J. Phononic crystals for liquid sensor applications. Measurement Science and Technology. 2009;20.

[14] Peng H, Pai PF, Deng H. Acoustic Multi-stopband Metamaterial Plates Design for Broadband Elastic Wave Absorption and Vibration Suppression. International Journal of Mechanical Sciences. 2015;103:104-14.

[15] Huang HH, Sun CT. Wave attenuation mechanism in an acoustic metamaterial with negative effective mass density. New Journal of Physics. 2009;11.

[16] Wang G, Wen XS, Wen JH, Shao LH, Liu YZ. Two-dimensional locally resonant phononic crystals with binary structures. Physical Review Letters. 2004;93.

[17] Liu Y, Su JY, Xu YL, Zhang XC. The influence of pore shapes on the band structures in phononic crystals with periodic distributed void pores. Ultrasonics. 2009;49:276-80.

[18] Soliman YM, Su MF, Leseman ZC, Reinke CM, El-Kady I, Olsson RH. Effects of release holes on microscale solid-solid phononic crystals. Applied Physics Letters. 2010;97. [19] Feng RX, Liu KX. Tuning the band-gap of phononic crystals with an initial stress. Physica B-Condensed Matter. 2012;407:2032-6. 
[20] Zhou X, Chen C. Tuning the locally resonant phononic band structures of twodimensional periodic electroactive composites. Physica B: Condensed Matter. 2013;431:2331.

[21] Wang YF, Wang YS, Su XX. Large bandgaps of two-dimensional phononic crystals with cross-like holes. Journal of Applied Physics. 2011;110.

[22] Phononic Crystals Fundamentals and Applications. In: Khelif A, Adibi A, editors. 1st ed: Springer; 2016.

[23] Lucklum R, Ke MZ, Zubtsov M. Two-dimensional phononic crystal sensor based on a cavity mode. Sensors And Actuators B-Chemical. 2012;171:271-7.

[24] Li FL, Wang YS, Zhang CZ, Yu GL. Bandgap calculations of two-dimensional solidfluid phononic crystals with the boundary element method. Wave Motion. 2013;50:525-41.

[25] Li E, He ZC, Jiang Y, Li B. 3D mass-redistributed finite element method in structuralacoustic interaction problems. Acta Mechanica. 2015:1-23.

[26] Li E, He ZC, Xu X, Liu GR. Hybrid smoothed finite element method for acoustic problems. Computer Methods in Applied Mechanics and Engineering. 2015;283:664-88.

[27] Zheng H, Zhang CZ, Wang YS, Sladek J, Sladek V. A meshfree local RBF collocation method for anti-plane transverse elastic wave propagation analysis in $2 \mathrm{D}$ phononic crystals. Journal of Computational Physics. 2016;305:997-1014.

[28] Li E, He ZC, Wang G, Liu GR. An efficient algorithm to analyze wave propagation in fluid/solid and solid/fluid phononic crystals. Computer Methods in Applied Mechanics and Engineering. 2018;333:421-42.

[29] Wang G, Wen JH, Liu YZ, Wen XS. Lumped-mass method for the study of band structure in two-dimensional phononic crystals. Physical Review B. 2004;69.

[30] Liu Y, Gao L-t. Explicit dynamic finite element method for band-structure calculations of 2D phononic crystals. Solid State Communications. 2007;144:89-93.

[31] Zhao JF, Li Y, Liu WK. Predicting band structure of 3D mechanical metamaterials with complex geometry via XFEM. Computational Mechanics. 2015;55:659-72.

[32] He ZC, Li E, Liu GR, Li GY, Cheng AG. A mass-redistributed finite element method (MR-FEM) for acoustic problems using triangular mesh. Journal of Computational Physics. 2016;323:149-70.

[33] Li E, He ZC, Wang G. An exact solution to compute the band gap in phononic crystals. Computational Materials Science. 2016;122:72-85.

[34] Li E, He ZC, Hu JY, Long XY. Volumetric locking issue with uncertainty in the design of locally resonant acoustic metamaterials. Computer Methods in Applied Mechanics and Engineering. 2017;324:128-48.

[35] Li E, He ZC, Wang G, Liu GR. An ultra-accurate numerical method in the design of liquid phononic crystals with hard inclusion. Computational Mechanics. 2017.

[36] Wang Y-F, Wang Y-S. Multiple wide complete bandgaps of two-dimensional phononic crystal slabs with cross-like holes. Journal of Sound and Vibration. 2013;332:2019-37.

[37] Ashcroft NW, Mermin ND. Solid state physics. New York: , Holt, 1976.

[38] Liu GR. Meshfree methods : moving beyond the finite element method. 2nd ed. Boca Raton: CRC Press,; 2010.

[39] Liu GR. A generalized gradient smoothing technique and the smoothed bilinear form for Galerkin formulation of a wide class of computational methods. International Journal of Computational Methods. 2008;05:199-236. 
[40] Liu GR. On G Space Theory. International Journal of Computational Methods. 2009;6:257-89.

[41] Liu GR. A G space theory and a weakened weak (W2) form for a unified formulation of compatible and incompatible methods: Part I theory. International Journal for Numerical Methods in Engineering. 2010;81:1093-126.

[42] Liu GR, Nguyen TT. Smoothed finite element methods. Boca Raton: Taylor \& Francis,; 2010. p. 691 p.

[43] Li E, Zhang Z, Chang CC, Zhou S, Liu GR, Li Q. A New Homogenization Formulation for Multifunctional Composites. International Journal of Computational Methods. 2016;13.

[44] Li E, He ZC, Xu X, Liu GR, Gu YT. A three-dimensional hybrid smoothed finite element method (H-SFEM) for nonlinear solid mechanics problems. Acta Mechanica. 2015;226:4223-45.

[45] Li E, He ZC. Development of a perfect match system in the improvement of eigenfrequencies of free vibration. Applied Mathematical Modelling. 2017;44:614-39.

[46] Liu GR. On Partitions of Unity Property of Nodal Shape Functions: Rigid-BodyMovement Reproduction and Mass Conservation. International Journal of Computational Methods. 2016;13:1640003. 